\title{
RIA: aplicación de una metodología para el seguimiento de la localización de los ODS
}

\section{Applying RIA methodology for SDGs monitoring at a local level}

\section{Martín Freigedo, I Alejandro Milanesi, ${ }^{I I}$ Carolina FerreiraIII}

I Doctor en Ciencias Sociales (Flacso-México). Máster en Políticas Públicas (Universidad Autónoma Metropolitana, México). Docente e investigador del Instituto de Ciencia Política, Facultad de Ciencias Sociales, Universidad de la República, Uruguay. Miembro del Sistema Nacional de Investigadores.

\·martin.freigedo@cienciassociales.edu.uy

https://orcid.org/0000-0003-0177-8845

II Doctor en Ciencia Política (Universidad de la República, Uruguay). Máster en Políticas Públicas (Victoria University of Wellington, Nueva Zelanda). Investigador y docente del Instituto de Ciencia Política, Facultad de Ciencias Sociales. Universidad de la República, Uruguay.

$\triangle \cdot$ alemc83@gmail.com

https://orcid.org/ 0000-0001-7338-0380

III Economista. Máster en Administración Pública (Harvard Kennedy School). Máster en Desarrollo Sostenible y Economía Social (Universidad de Huelva, España) y Montesquieu IV (Burdeos, Francia). Diploma en Cooperación Internacional y Desarrollo (Universidad CLAEH, Uruguay). Docente en la Universidad Católica del Uruguay y la Facultad de Ciencias Económicas y Administración de la Universidad de la Republica.

$\varangle \cdot$ caroferreiratbo@gmail.com

https://orcid.org/0000-0002-6100-6508

RECIBIDO: 15.9 .2019

ACEPTADO: 9.11 .2019

\section{Resumen}

La Agenda 2030 de Desarrollo Sostenible y los ODS son actualmente el eje central de acción para los organismos internacionales y fijan buena parte de las acciones de desarrollo y cooperación a nivel global. En el plano técnico implica un esfuerzo importante de construcción y sistematización de información para la alineación y posterior monitoreo de políticas e indicadores. Especialmente en el nivel subnacional, esto contrasta 
con gobiernos locales pequeños, a menudo con poca experiencia y pocos recursos dedicados al monitoreo de políticas, lo que afecta la capacidad para conocer su desempeño respecto de los ODS. Este artículo presenta la adaptación de una metodología de alineación de los ODS a escala local y su aplicación empírica a seis gobiernos departamentales de Uruguay. Su aplicación permite conocer el aporte de estos gobiernos al cumplimiento de la Agenda 2030 y profundizar en debates respecto de las competencias y calidad de las políticas en los niveles subnacionales.

Palabras clave: desarrollo sostenible, administración local, Uruguay.

\section{Abstract}

The 2030 Agenda for Sustainable Development and the SDGs are currently a central pillar of action for international organizations, determining development and cooperation actions at a global scope. From a technical perspective, to build and systematize information for the alignment and subsequent monitoring of policies and indicators implies a significant effort. Especially at the sub-national level, this collides with small local governments often lacking experience and resources when it comes to monitoring policies, which affects their ability to keep track of their performance towards SDGs. This article presents the adaptation of RIA methodology for SDGs monitoring at a local level. Its empirical application in six departmental governments in Uruguay made it possible to assess their contributions to the fulfillment of the 2030 Agenda and to deepen debates regarding the prerogatives and quality of policies at sub-national levels.

Keywords: sustainable development, local government, Uruguay.

\section{Introducción 1}

La Agenda 2030 de Desarrollo Sostenible es un compromiso aprobado por todos los países miembros de la Asamblea General de las Naciones Unidas el 25 de setiembre de 2015. Se trata de un intento de construcción de una estrategia viable en el plano social, económico y ambiental con el principal desafío para los Estados de erradicación de la pobreza. Esta agenda es actualmente uno de los ejes centrales de acción para los organismos internacionales y fija buena parte de los esfuerzos de desarrollo y cooperación

1 Este artículo fue elaborado con base en la información recabada en el convenio para la Localización de los Objetivos de Desarrollo Sostenible en el Uruguay, ejecutado en el período 2018-2019 entre la Oficina de Planeamiento y Presupuesto de Presidencia de la República (OPP), el Programa de las Naciones Unidades para el Desarrollo (PNUD) y el Centro de Informaciones y Estudios del Uruguay (CIESU). 
a nivel global. Su faceta más conocida es la elaboración de los 17 Objetivos de Desarrollo Sostenible (ODS), los cuales contienen 169 metas y 231 indicadores de seguimiento. ${ }^{2}$

En el plano técnico, la adopción de la Agenda 2030 implica un esfuerzo importante de construcción y sistematización de información para el monitoreo de políticas e indicadores; más concretamente, la generación de información confiable y adaptada a los contextos específicos que permitan monitorear avances y brechas en el tránsito hacia el cumplimiento de los ODS, así como la consiguiente rendición de cuentas.

En ese marco, se han desarrollado múltiples guías y documentos para ayudar a los gobiernos a monitorear y evaluar el cumplimiento de los ODS en sus países, tanto para toda la agenda como en temáticas específicas (Schmidt-Traub et al., 2017; Kanuri et al., 2016; Sustainable Development Solutions Network, 2015; United Nations Development Programme [UNDP], 2017a; Global Taskforce of Local and Regional Governments, 2016; Organisation for Economic Co-operation and Development [OECD], 2020). A partir de estas múltiples guías se han generado distintas formas y enfoques para presentar la información, desde reportes nacionales de monitoreo formales de los indicadores hasta la aplicación de novedosas metodologías o toolkits. Aunque han florecido distintas metodologías para el seguimiento de los ODS (Reyers et al., 2017), la mayoría de estas son muy complejas y orientadas al nivel nacional (Liu et al., 2019). Requieren potentes sistemas de información, datos desagregados por región y equipos técnicos calificados para su aplicación. Especialmente en el nivel subnacional, esto choca con gobiernos locales pequeños, muchas veces con poca experiencia y recursos dedicados al monitoreo de políticas. Por tanto, esto afecta la capacidad de los gobiernos locales para monitorear su desempeño respecto de los ODS y fijarse estrategias de desarrollo asociadas a la Agenda 2030.

Este artículo busca hacer dos aportes principales. En primer lugar, pretende presentar la adaptación de una metodología de seguimiento de los ODS a escala local: el modelo de evaluación rápida integrada (RIA, por sus siglas en inglés). Este modelo permite a los gobiernos locales contar con una línea de base del aporte de sus políticas a los ODS para analizar estrategias de políticas y diseñar prácticas de generación de información más profundas.

En segundo lugar, tiene como objetivo la aplicación empírica de esta metodología en seis departamentos de Uruguay. Su aplicación no sólo permitió conocer el aporte de estos gobiernos al cumplimiento de la Agenda 2030 sino presentar información empírica respecto a debates más amplios presentes en el estudio de los gobiernos subnacionales. En particular aquellos relacionados al incremento de competencias y áreas de intervención de los niveles subnacionales en las políticas públicas. Un punto central que se debe aclarar es que el alto grado de centralidad del Estado uruguayo hace que históricamente los 
gobiernos subnacionales carezcan de autonomía a la hora de la gestión, sobre todo desde el punto de vista normativo (Freigedo y Goinheix, 2019). En este sentido, el artículo demuestra que las acciones de los gobiernos departamentales están orientadas a los ODS asociados a infraestructura y ciudades. Sin embargo, también es alto el porcentaje de acciones alineadas a ODS en que estos gobiernos no tienen funciones tan claramente definidas desde el punto de vista normativo, dada la ambigüedad de las competencias establecidas por ley, asociadas sobre todo a políticas sociales como educación y salud.

Para este cometido, el artículo se estructura de la siguiente forma. En un primer apartado se presentan alguas consideraciones básicas sobre la relevancia de la localización de los ODS. El segundo se centra en la presentación de la metodología específica (RIA) y la adaptación realizada. En el tercer apartado se presentan los resultados de la sistematización para el caso de los gobiernos departamentales en Uruguay. Finalmente, en las conclusiones se presenta un conjunto de desafíos para avanzar en la agenda de localización de los ODS a partir de la contrucción de nuevas herramientas metodológicas de monitoreo y evaluación.

\section{Localización de los ODS y seguimiento de políticas}

El logro de los ODS está, aunque no únicamente, condicionado por la capacidad de los gobiernos para desarrollar estrategias de desarrollo efectivas. Esto implica el involucramiento no sólo de los gobiernos nacionales sino también de los niveles subnacionales (Sianes y Vela-Jiménez, 2020). El proceso de seguimiento y monitoreo de los ODS en los territorios ha sido definido como localización. Esta puede ser entendida como un proceso que implica adaptar, implementar y monitorear los ODS a nivel local (Kanuri et al., 2016, p. 15). A su vez: «[...] tiene en cuenta los contextos subnacionales en el logro de la Agenda 2030, desde el establecimiento de los objetivos y metas, hasta la determinación de los medios de implementación, y el uso de indicadores para medir y monitorear el progreso hecho» (Global Taskforce of Local and Regional Governments, 2016 p. 5).

Se asume que los gobiernos subnacionales son actores centrales en la implementación de la Agenda 2030 en tanto buena parte de sus competencias y prestación de servicios están directamente vinculadas a aspectos ambientales, urbanos, económicos y sociales de sus territorios (Patole, 2018; United Cities and Local Governments [UCLG], 2015, 2019). Así, por ejemplo, se ha calculado que el 65\% de las metas de los ODS podrían estar en riesgo de alcanzarse si no se involucra claramente a los gobiernos locales (UCLG, 2019). En los países de la OCDE son responsables de aproximadamente el 60\% de toda la inversión pública y $40 \%$ del gasto público, lo que incluye aspectos críticos como el cambio climático (OECD, 2020). 
La localización de los ODS apunta a funcionar como un acelerador de la implementación de la Agenda 2030 (Oosterhof, 2018). Se destaca además la necesidad de generar información a nivel local que permita comprender las necesidades y prioridades de los territorios, para luego, sobre esa base, atender eventuales disparidades o inequidades territoriales en las estrategias de desarrollo. Asociado a esto, la cercanía y el mayor conocimiento de la realidad territorial hace que los gobiernos subnacionales sean actores privilegiados para generar procesos y canales institucionales de participación ciudadana y rendición de cuentas (Selee y Peruzzotti, 2009).

Finalmente, la relevancia de la localización de los ODS se sustenta en que los gobiernos subnacionales presentan buenas prácticas muchas veces invisibilizadas como consecuencia de la falta de sistematización de la información y de la falta de estrategias de comunicación. La localización de los ODS apunta justamente a proveer evidencia y con ello fomentar también estrategias más efectivas y coordinadas de desarrollo entre los niveles de gobierno.

En ese marco, Uruguay ha hecho avances importantes en materia de localización de los ODS a partir de la aplicación de diversas metodologías (Oficina de Planeamiento y Presupuesto [OPP], 2019). Entre estas se cuentan enfoques participativos o consultivos con ciudadanos, identificación de problemas complejos con actores claves de la sociedad civil (trabajadores, empresarios, organizaciones sociales) e identificación de buenas prácticas de desarrollo en temas como medioambiente y hábitat, entre otros. ${ }^{3}$ La figura 1 resume la estrategia adoptada por el gobierno uruguayo, que estuvo centrada en cuatro modalidades diferentes de monitoreo.

Figura 1. Metodologías de localización de los ODS en Uruguay

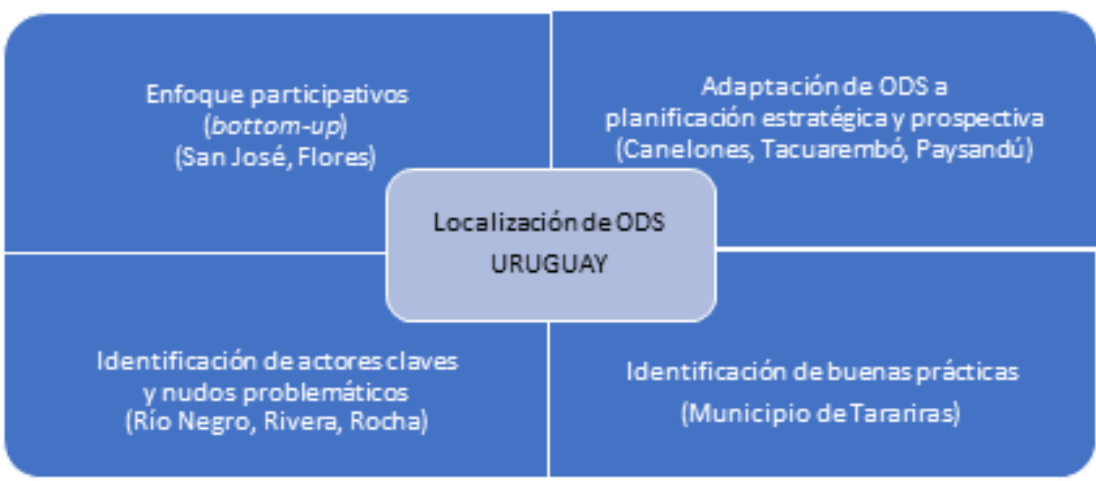

Fuente: Elaboración propia basada en Informe Voluntario 2019. 
De esta forma, las cuatro metodologías utilizadas fueron:

1. Enfoque participativo (bottom-up). En este caso el enfoque se basó en el trabajo consultivo con actores claves del territorio, del sector privado y organizaciones sociales de vecinos. Estas instancias permitieron trabajar sobre asuntos y problemas compartidos por los distintos grupos. Esta metodología no se enfocó tanto de trabajar en una asociación directa con los ODS sino a partir de comprender las problemáticas complejas y su vínculo con grupos de la población o localidades específicas.

2. Identificación de actores claves y nudos problemáticos. Esta metodología se basó en la realización de encuentros y entrevistas con actores claves de los territorios -empresarios, líderes sindicales, representantes de agencias de desarrollo, entre otros - para trabajar de forma más profunda sobre la identificación de nudos que limitan el desarrollo del departamento en sus distintas dimensiones. Esta metodología permite indagar con mayor detalle no sólo en la definición de los asuntos claves de la localidad sino en las raíces y las consecuencias para el sector productivo.

3. Adaptación de ODS a procesos de planificación y/o prospectivos. Esta metodología implicó un esfuerzo por vincular procesos de planificación y prospectivos en curso o ya realizados por los gobiernos departamentales a los ODS. Este enfoque tiene un impacto importante no sólo en ampliar el nivel de conocimiento sobre los ODS en las organizaciones públicas sino también internalizar la Agenda 2030 en los procesos de gestión.

4. Identificación de buenas prácticas a nivel local. Este enfoque buscó trabajar sobre la visibilización de experiencias locales de impacto para el cumplimiento de la Agenda 2030.

Estas estrategias permiten aproximarse desde diferentes abordajes al mismo objeto y tienden a ser complementarias. En este artículo nos centramos en el RIA como una metodología para el monitoreo de los ODS a nivel local y su aplicación práctica en seis gobiernos departamentales. En el siguiente apartado se presentan algunas características del RIA y su adaptación para el caso uruguayo.

\section{El RIA: metodología y adaptación al nivel local}

El RIA tiene como objetivo realizar un mapeo de los ODS (sus metas y objetivos) en relación con las prioridades nacionales y subnacionales a partir de la revisión de planes, agendas, políticas y otros documentos oficiales que delineen las estrategias de los 
gobiernos (UNDP, 2017b). Ello permite definir en qué medida los ODS están reflejados en las acciones de las organizaciones públicas, identificando líneas para su integración, así como posibles brechas en asuntos no adecuadamente abordados. Esta herramienta ha sido utilizada en más de diez países. No obstante, las experiencias subnacionales son mucho menores en cantidad por lo cual este caso representó una oportunidad de aplicación a escala local. La herramienta del RIA fue a su vez adaptada identificando tres categorías que definen las acciones implementadas por el gobierno departamental: planes, proyectos o acciones. Ello obedeció a la necesidad de identificar acciones que son distintas en términos de alcance de los objetivos, recursos destinados y temporalidad:

- Planes: iniciativas de largo alcance y que normalmente contemplan un conjunto de proyectos y acciones. Los planes suelen contar con documentos específicos que contienen objetivos, metas, plazos de ejecución, etc. Ejemplo de ellos son los planes de obra o los planes de ordenamiento territorial.

- Políticas: son iniciativas de mediano alcance. Pueden estar comprendidas en planes o funcionar de manera independiente. A diferencia de los planes, sus objetivos están normalmente limitados a un rango concreto de temas. Ejemplos de ello es la instalación de un servicio, programas o proyectos en coordinación con otras entidades.

- Acciones: refiere a iniciativas puntuales. Pueden estar comprendidas en planes o funcionar de manera independiente. Ejemplos de ellos son cursos, asistencias técnicas, promoción de prácticas, manteamientos o reparaciones.

Esta distinción entre diferentes alcances que tienen las iniciativas es una adaptación propia que se elaboró particularmente para el caso uruguayo y que puede ser replicada para otros casos.

\section{ODS y gobiernos departamentales}

El siguiente capítulo presenta la información de alineamiento de las iniciativas de los gobiernos departamentales en relación con los ODS. Los datos se basan en el relevamiento de seis departamentos: Paysandú, Rivera, Rocha, San José, Soriano y Tacuarembó. El criterio de selección estuvo centrado en abordar departamentos que representaran diferentes regiones del país y que contaran con características disímiles. El relevamiento se realizó mediante una recolección de información que implicó dos estrategias diferentes. En primer lugar, se realizó un análisis de información secundaria a partir de los sistemas de información existentes (Mirador Ciudadano de OPP, el Sistema 
de Información Territorial de la Dirección Nacional de Ordenamiento Territorial). En segundo lugar, se realizó un trabajo conjunto entre el equipo de Centro de Informaciones y Estudios del Uruguay (CIESU) y las autoridades departamentales (se trabajó con todos los directores departamentales y sus equipos). En estos encuentros se sistematizaron las iniciativas que llevan adelante los gobiernos departamentales y se clasificaron según su alcance en planes, políticas o acciones.

El relevamiento permitió identificar 1096 vinculaciones entre las actividades de los gobiernos departamentales y los 17 ODS. Hacemos referencia a vinculaciones en tanto no se trata de una categorización o correspondencia única. Cada iniciativa de los gobiernos departamentales puede estar asociada a más de un ODS. De la misma forma, algunas iniciativas departamentales pueden incluir varias acciones, como los planes o políticas (véase gráfico 1).

Gráfico 1. Alineación de las iniciativas de los gobiernos departamentales a los ODS

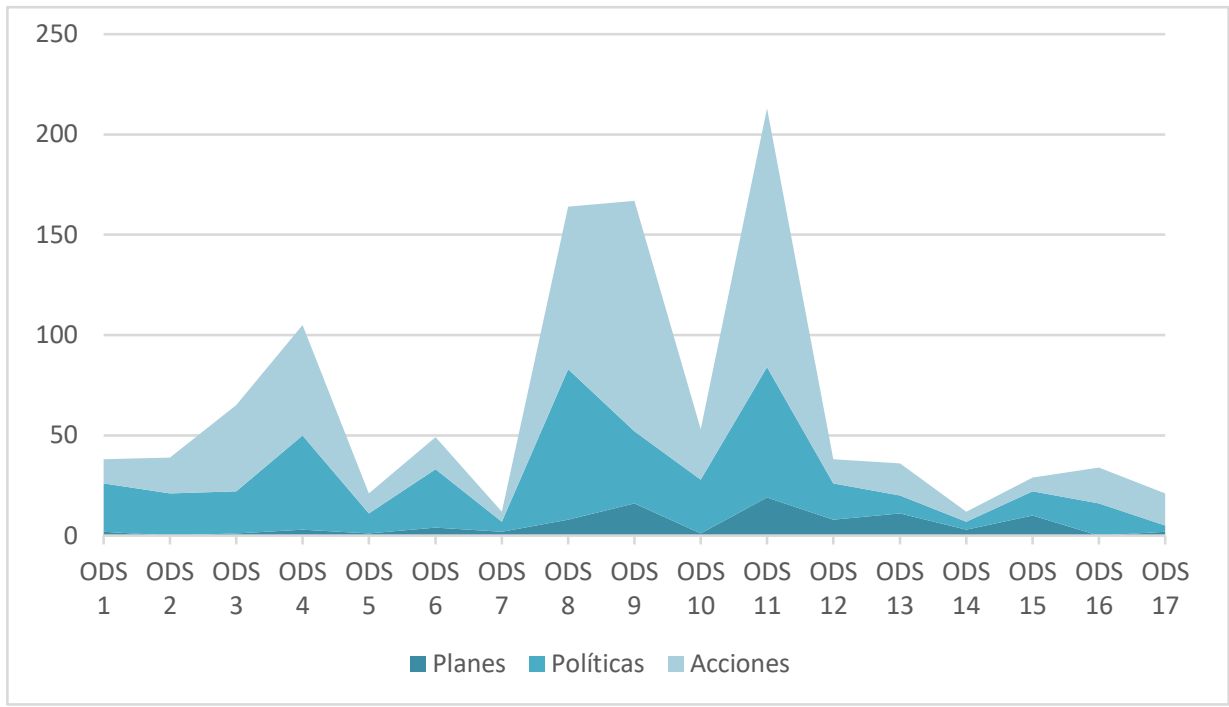

Fuente: Informe Nacional Voluntario 2019.

Los datos recabados muestran que todos los ODS están presentes en las actividades de los gobiernos departamentales. Ello da cuenta de la amplitud de funciones que estos gobiernos cumplen en la gestión de políticas públicas. No obstante, no todos los ODS están presentes en la misma magnitud. El mayor porcentaje de vinculaciones se encuentra en el ODS 11 correspondiente a ciudades y comunidades sostenibles con un $19 \%$ del total de vinculaciones. 
En este ODS se destacan intervenciones relacionadas con el mejoramiento de calles, caminería y plazas, así como iniciativas de mayor integralidad como la urbanización de algunos barrios marginales. Este ODS también se relaciona con el mejoramiento del transporte urbano y su sostenibilidad. Los gobiernos departamentales han tenido históricamente un rol central en este cometido, destacándose acciones que no solo apuntan a la mejora de la infraestructura y logística del transporte, sino también con intervenciones orientadas a la educación vial. En la misma línea van las acciones que se desarrollan en relación con la prevención de desastres. Los gobiernos departamentales participan activamente en los espacios interinstitucionales denominados comités de emergencia que operan en todos los territorios. También comprendido en el ODS 11 se encuentran las funciones relacionadas con la gestión de residuos, pues varios departamentos han impulsado importantes proyectos para mejorar los sitios de disposición final y planes de reciclaje.

En segundo lugar se encuentran los ODS 9 y 8, referentes a la industria, innovación e infraestructura, y el crecimiento económico y empleo sostenido y sostenible, ambos con un $15 \%$ de las vinculaciones. El ODS 9 forma parte del rol que cumplen los gobiernos departamentales en la inversión de infraestructura, la cual ha tendido a ser cada vez más relevante (Freigedo y Rodríguez, 2019). También, a nivel local, los gobiernos han colaborado en el fortalecimiento de pequeñas y medianas empresas, mediante capacitaciones, acceso a créditos y fomento de la inversión.

En cuanto al ODS 8, los gobiernos departamentales han desarrollado un abanico de políticas de capacitación en áreas estratégicas según el caso y la región, buena parte de ellas dedicadas a incorporar jóvenes y mujeres al mercado laboral. Asimismo, una de las metas de este objetivo pasa por promover un turismo sostenible. Las intervenciones, generalmente en colaboración con el Gobierno nacional, también han sido diversas, desde acciones específicas como la formación y concientización entre la población para promover este tipo de turismo, hasta planes de turismo social y comunitario que abarcan todo el territorio departamental.

El ODS 4, enfocado en promover una educación de calidad, representa el 10\% de las vinculaciones. Si bien los gobiernos departamentales no tienen competencias en la educación formal, realizan diversas intervenciones para el mantenimiento edilicio de la infraestructura educativa, o bien desarrollando cursos de educación no formal sobre temas específicos demandados por la ciudadanía, la mayor parte de las veces orientados a la adquisición de herramientas para el mercado laboral. También brindan una oferta diversificada en algunas áreas referidas a cultura y recreación. Por último, estos gobiernos han sido tradicionalmente actores fundamentales a la hora de brindar apoyos a jóvenes que pretenden continuar su formación por medio de becas o residencias universitarias en Montevideo (Midaglia y Castllo, 2010). 
En total los cuatro ODS mencionados representan un $60 \%$ de las vinculaciones. Vale destacar, no obstante, algunas intervenciones puntuales en otros ODS. Especialmente, las referidas a los planes de ordenamiento territorial, tanto a nivel departamental como local, que son intervenciones que contribuyen a los ODS referidos a los asuntos ambientales (ODS 13, 14 y 15).

Si consideramos las iniciativas de los gobiernos departamentales de acuerdo con su distinción en planes, políticas y acciones es posible notar que la presencia de algún tipo de plan está presente en todos los ODS con excepción de los ODS 2 y 16. Los planes se encuentran mayormente presentes en los ODS 11 y 9 (con un $21 \%$ y 18\%, respectivamente). Esto parece razonable con el hecho de que se trata de los ODS más directamente vinculados a las competencias históricas y los presupuestos de los gobiernos departamentales relacionados con el mantenimiento de la infraestructura urbana y vial o el transporte. Este tipo de iniciativas se reflejan frecuentemente en detallados planes de obra, tanto para las capitales como para localidades más pequeñas del departamento. Debe destacarse también la cantidad de planes vinculados a ODS referidos a la dimensión ambiental, como los son el ODS 13 de acción por el clima, y el ODS 15 referido a la vida de ecosistemas terrestres, con un $12 \%$ y $11 \%$ del total de los planes, respectivamente. Este dato sugiere un incremento de la relevancia de los temas ambientales en la agenda de los gobiernos departamentales y el consiguiente desarrollo de iniciativas de mayor porte. La elaboración de planes en estas arenas de políticas está relacionada directamente con la aprobación de la Ley de Ordenamiento Territorial y Desarrollo Sostenible, que ha incentivado la creación de diferentes instrumentos de ordenamiento territorial.

En cuanto a las políticas se destaca el ODS 8 (trabajo decente y crecimiento económico) y ODS 11 , con un $18 \%$ y $15 \%$, respectivamente. Por su parte, el ODS 4 (educación de calidad) representa un $11 \%$ del total de las vinculaciones de las políticas. Con un porcentaje menor están representados los ODS 6 (agua limpia y saneamiento) con un 7\%, y luego los ODS 1 (fin de la pobreza), 2 (hambre cero) y 3 (salud y bienestar) vinculados a la dimensión social de los objetivos, con un $5 \%$ cada uno de ellos. Finalmente, las acciones constituyen las iniciativas presentes en mayor cantidad. Se trata de intervenciones de corto plazo que los gobiernos desarrollan de forma puntual o para atender objetivos de menor porte. Nuevamente aquí los ODS 9 y 11 se destacan como los más frecuentes. No obstante, es interesante ver una alta presencia de los ODS 8, 4 y 3, que en total representan el $31 \%$ de las acciones. Los datos sobre políticas y acciones no solo reafirman la multiplicidad de funciones de los gobiernos departamentales, sino también el desdoblamiento hacia intervenciones menos tradicionales o alejadas de sus competencias (Freigedo y Goinheix, 2019). De todas formas, estas acciones también dan cuenta de que los gobiernos departamentales actúan sobre estas arenas de políticas con una planificación menor que en otras áreas más tradicionales. Esto puede tener que ver con 
un menor desarrollo de capacidades desde los propios gobiernos y, en muchos casos, con una menor coordinación con el nivel central (Munielo-Gallo y Rodríguez, 2014).

\section{Discusión y conclusiones}

Este artículo presenta dos novedades, una metodológica y otra empírica. En relación con lo metodológico, presenta una adaptación de un mecanismo de seguimiento relevante para la alineación de los ODS, el RIA. En este sentido, la distinción entre planes, políticas y acciones permite calibrar mejor el alcance de las intervenciones de los gobiernos sobre la Agenda 2030. Como se mencionó en el trabajo, la distinción permite observar el grado de planificación que llevan adelante los gobiernos de acuerdo con los diferentes tipos de intervención. A su vez, es una de las primeras experiencias a nivel mundial de aplicación de la metodología a nivel local, ya que la mayoría de los ejemplos responden al nivel nacional. En este sentido, es un insumo para futuros trabajos que busquen replicar la metodología de alineación de los ODS a nivel local.

En relación con lo empírico, la aplicación sobre el caso uruguayo confirma una serie de hallazgos importantes. En primer lugar, diversos estudios han señalado que los gobiernos departamentales en Uruguay han incrementado su rango de intervención en políticas a partir de un mayor despliegue en políticas sociales, ambientales y de desarrollo productivo (Freigedo y Rodriguez, 2019). Concomitantemente, han ampliado también su agenda de cooperación internacional y desarrollo, sobre la base de un trabajo conjunto entre el gobierno nacional y la intervención de programas internacionales, particularmente del PNUD, con una agenda conjunta de trabajo sobre diferentes aristas que afectan el desarrollo del territorio. Este artículo aporta evidencia empírica a este argumento. Los gobiernos departamentales hoy tienen un abanico de intervenciones que cubren buena parte de la agenda temática de políticas. Tomando en cuenta la información presentada anteriormente, los gobiernos departamentales tienen intervenciones, en mayor o menor medida, en todos los ODS.

En segundo lugar, la evidencia presentada aporta también elementos de debate respecto de las características de las políticas desarrolladas por los gobiernos departamentales. El RIA mostró que las intendencias desarrollan una gran cantidad de acciones, pero no siempre bajo un marco de planificación global. Por el contrario, suelen prevalecer acciones puntuales o políticas de corto alcance, como puede apreciarse en algunos ODS donde la presencia de planes es escasa o nula, sobre todo en aquellos vinculados a los sectores de políticas sociales más clásicas.

Este impulso en el desarrollo de nuevas políticas en el ámbito social no parece ir acompañado aún de estrategias más planificadas como planes o agendas sociales del departamento. En este sentido, un desafío para la gestión departamental pasa por la 
planificación en estas arenas de políticas, con el objetivo de optimizar recursos y no duplicar acciones con otras organizaciones del nivel central que también intervienen allí.

Asimismo, es importante señalar que este primer paso de localización, asociado a la alineación de los ODS, es de relevancia para continuar el camino. En este sentido, se debe pensar en la planificación de un sistema de monitoreo que permita avanzar hacia el cumplimiento de los ODS a nivel subnacional, basado en la asociación de las metas e indicadores que presenta la agenda pero de acuerdo con la realidad de cada territorio. Esto entraña un esfuerzo de adaptación al contexto local que va más allá del monitoreo de las metas e indicadores globales. A su vez, conlleva necesariamente una discusión local respecto de la mejor forma de integrar y dar sentido a la Agenda 2030 en los territorios. En este sentido, implica la necesidad de desarrollar capacidades locales de implementación, acceso a financiamiento y monitoreo de políticas, una dimensión frecuentemente débil en los gobiernos subnacionales.

Finalmente, como señala el informe GOLD V de la CGLU (2020), cinco años después de comenzada la implementación y pese a avances importantes, el desarrollo de la Agenda 2030 todavía tiene un camino importante por recorrer. La Agenda 2030 puede constituirse en una gran oportunidad desde lo local para crear una nueva generación de políticas, donde lo económico, lo social y lo ambiental vayan de la mano para la reactivación productiva de las regiones, la generación de empleo y la protección social y ambiental de las comunidades.

\section{Referencias bibliográficas}

Ciudades y Gobiernos Locales Unidos. (2020). La localización de las agendas mundiales: Cómo la acción local transforma las ciudades y territorios. Recuperado de https://www.gold.uclg.org/sites/default/files/SP-GOLD\%20V-WEB.pdf.

Freigedo, M., y Goinheix, S. (2019). Análisis del nivel de autonomía de los gobiernos departamentales en Uruguay. En A. Cardarello y P. Ferla (coords.), Descentralización en Uruguay: Propuestas para avanzar en la agenda (pp. 13-31). Montevideo: Fundación Konrad Adenauer.

Freigedo, M., y Rodríguez, J. (2019). Las capacidades estatales de los gobiernos departamentales en Uruguay. En E. J. Grin, E. Completa, A. P. Carrera-Hernández y F. L. Abrucio (coords), Capacidades estatales en gobiernos locales iberoamericanos: actualidad, brechas y perspectivas. Río de Janeiro: Fundación Getulio Vargas.

Global Taskforce of Local and Regional Governments. (2016). Guía para la localización de los ODS: Implementación y monitoreo subnacional. Recuperado de https:// www.gub.uy/agencia-uruguaya-cooperacion-internacional/comunicación/publicaciones/guia-para-localizacion-ods-implementacion-monitoreo-subnacional. 
Kanuri, C., Revi, A., Espey, J., y Kuhle, H. (2016). Cómo implementar los ODS en las ciudades: Un manual introductorio para quienes trabajan en el ámbito del desarrollo urbano sostenible. Recuperado de https://reds-sdsn.es/wp-content/uploads/2018/ 12/SDG-Cities-Guide-Spanish_edited_Final-version.pdf.

Liu, S., Bai, J., y Chen, J. (2019). Measuring SDG 15 at the county scale: Localization and practice of SDGs indicators based on geospatial information. International Journal of Geo-Information, 8(11), 515.

Midaglia, C., y Castillo, M. (2010). La oferta publica social en el Uruguay 2005-2009: Informe final del convenio con el Instituto de Ciencia Política. Montevideo: MIDES. (Uruguay Social, 4).

Muinelo-Gallo, L., y Rodríguez Miranda, A. (2014). Descentralización fiscal, calidad de gestión de gobierno y disparidades regionales en Uruguay. Estudios de Economía, 41(2), 219-250.

Oficina de Planeamiento y Presupuesto. (2019). Informe nacional voluntario. Uruguay 2019. Montevideo: OPP.

Oosterhof, P. D. (2018). Localizing the sustainable development goals to accelerate implementation of the 2030 agenda for sustainable development. The Governance Brief, 33, 1-14.

Organisation for Economic Co-operation and Development. (2020). A territorial approach to the sustainable development goals: Synthesis report. París: OECD.

Patole, M. (2018). Localization of SDGs through disaggregation of KPIs. Economies, $6(1), 15$.

Reyers, B., Stafford-Smith, M., Erb, K. H., Scholes, R. J., y Selomane, O. (2017). Essential variables help to focus sustainable development goals monitoring. Current Opinion in Environmental Sustainability, 26-27, 97-105.

Schmidt-Traub, G., Kroll, C., Teksoz, K., Durand-Delacre, D., y Sachs, J. D. (2017). National baselines for the sustainable development goals assessed in the SDG index and dashboards. Nature Geoscience, 10(8), 547-555.

Selee, A., y Peruzzotti, E. (2009) Participatory innovation and representative democracy in Latin America. Baltimore: John Hopkins University Press.

Sianes, A., y Vela-Jiménez, R. (2020). Can differing opinions hinder partnerships for the localization of the sustainable development goals? Evidence from marginalized urban areas in Andalusia. Sustainability, 12(14), 5797.

Sustainable Development Solutions Network, (2015). Indicators and a monitoring framework for the sustainable development goals - launching a data revolution for the SDGs. Recuperado de: https://resources.unsdsn.org/indicators-and-a-monitoring-framework-for-sustainable-development-goals-launching-a-data-revolution-for-the-sdgs. 
United Cities and Local Governments (2015). The sustainable development goals: What local governments need to know. Recuperado de https://www.uclg.org/sites/default/files/the_sdgs_what_localgov_need_to_know_0.pdf.

United Cities and Local Governments. (2019). Towards the localization of the SDGs: $3 r d$. report. Recuperado de https://www.uclg.org/sites/default/files/towards_the_localization_of_the_sdgs_0.pdf.

United Nations Development Programme. (2017a). Institutional and coordination mechanisms. guidance note on facilitating integration and coherence for SDG implementation. Recuperado de http://www.undp.org/content/undp/en/home/librarypage/sustainable-development-goals/institutional-and-coordinationmechanisms---guidance-note.html.

United Nations Development Programme. (2017b). Rapid integrated assessment (RIA) facilitating mainstreaming of SDGs into national and local plans. Recuperado de https://www.undp.org/content/undp/en/home/librarypage/sustainable-development-goals/rapid-integrated-assessment---mainstreaming-sdgs-into-nationala.html. 